\title{
Clinicians' Perspectives on Barriers and Enablers of Optimal Prescribing in Patients with Dementia and Coexisting Conditions
}

\author{
Ariel R. Green, MD, MPH, Patricia Lee, BA, Emily Reeve, PhD, Jennifer L. Wolff, PhD, \\ Chi Chiung Grace Chen, MD, MHS, Rachel Kruzan, MD, and \\ Cynthia M. Boyd, MD, MPH
}

Purpose: Patients with dementia experience high rates of polypharmacy, potentially inappropriate medication use, and adverse drug events. There is little guidance for clinicians on how to optimize prescribing for this population. Our objective was to investigate clinician-perceived barriers to and facilitators of optimizing prescribing for people with dementia.

Methods: Qualitative study involving semistructured interviews of primary care and specialist clinicians in urban, suburban, and rural settings. Transcripts were analyzed using qualitative content analysis.

Results: Interviews were conducted with 12 primary care and 9 specialist clinicians, with a mean (SD) age of 47 (9) and mean (SD) of 14 (10) years in practice. Clinicians cited decisions regarding the following drug classes as particularly challenging: oral anticoagulants, antidiabetic agents, statins, bladder antimuscarinics, and antipsychotics. Perceived enablers of optimizing prescribing included access to interdisciplinary services and guidelines for nondementia illnesses (eg, diabetes) addressing the care of people with dementia. Barriers included the lack of data on efficacy and safety of most medications in people with dementia, difficulty assessing medication effects in an individual patient, and the perception that stopping medications is seen as "giving up." Clinicians used a variety of strategies to discuss risks and benefits of medications with patients and caregivers.

Conclusions: Clinicians identified numerous barriers to and some facilitators of optimizing prescribing in people with dementia. More data are needed on the benefits and harms of stopping medications in this population. Research should also test different approaches for supporting informed decision making about medications by people with dementia and caregivers. (J Am Board Fam Med 2019;32: 383-391.)

Keywords: Adverse Decision Making, Drug Reaction, Comorbidity, Dementia, Geriatrics, Polypharmacy, Potentially Inappropriate Medications, Primary Health Care, Qualitative Research, Risk Assessment

Dementia affects 5.4 million people in the United States. ${ }^{1}$ People with dementia, caregivers, and clinicians face numerous decisions about using medications to prevent or treat coexisting conditions and to alleviate symptoms, such as pain, insomnia, incontinence, and agitation. These decisions carry trade-offs between quality and length of life or

This article was externally peer reviewed.

Submitted 8 November 2018; revised 29 January 2019; accepted 30 January 2019.

From Division of Geriatric Medicine and Gerontology, Johns Hopkins University School of Medicine, Baltimore, MD (ARG, CMB); University of Illinois College of Medicine at Chicago, Chicago (PL); Kolling Institute of Medical Research, Northern Clinical School, Faculty of Medicine, between different dimensions of quality of life (for example, medications to treat pain may cause sedation). ${ }^{2}$ Optimal prescribing for older adults with dementia involves prescribing medications that will help people achieve their goals of care and deprescribing, or withdrawing, potentially inappropriate

The University of Sydney, Sydney, Australia (ER); Department of Health Policy and Management, Johns Hopkins University Bloomberg School of Public Health, Baltimore, MD (JLW); Department of Gynecology and Obstetrics, Johns Hopkins University School of Medicine (CCGC); Johns Hopkins Community Physicians, Baltimore, MD (RK); Department of Health Policy and Management, Baltimore, Johns Hopkins University Bloomberg School of Public Health, MD (CMB). 
medications (PIMs), in which harms of medications may outweigh benefits.

Most people with dementia have multiple chronic conditions. ${ }^{3}$ Achieving optimal medication use for this population is challenging, ${ }^{4}$ as evidenced by high rates of polypharmacy and PIM use. ${ }^{5}$ People with dementia take 5 to 10 medications, on average, of which 1 to 2 are prescribed for dementia and the remainder for coexisting conditions. ${ }^{5}$ The prevalence of PIM use among people with dementia ranges from $15 \%$ to $38 \%{ }^{6}$ Given the prolonged and variable time course of dementia, determining whether a medication to prevent or treat a coexisting condition is likely to result in benefits during the person's remaining lifespan is difficult. Medications that were once appropriate may become inappropriate as dementia progresses; exactly when may be unclear.

Little is known about how clinicians make prescribing decisions for people with dementia. To begin developing a framework to optimize prescribing decisions for this population, our objective was to investigate clinician-perceived barriers and facilitators of reducing polypharmacy and PIM use in people with dementia.

\section{Methods}

\section{Design and Study Setting}

The study design was based on semistructured interviews with primary care and specialist clinicians. Data collection took place from March 2017 to February 2018. The project was approved by the Johns Hopkins School of Medicine Institutional Review Board. Participants provided oral informed consent and received a $\$ 100$ gift card.

\section{Participants}

To obtain a wide range of perspectives and experiences, ${ }^{7}$ we sought physicians, certified registered nurse practitioners, and physician assistants who

Funding: This work was supported by K23AG054742 (Green) and K24AG056578 (Boyd) from the National Institute on Aging. Support was also provided by the Medical Student Training in Aging Research (MSTAR) program (NIA T35AG026758) for Ms. Lee's time.

Conflict of interest: Dr. Boyd writes a chapter on multimorbidity for UpToDate, for which she receives a royalty. ARG, PL, ER, JLW, CCGC, and RK declare that they have no conflicts of interest relevant to the content of this study.

Corresponding author: Ariel R. Green, MD, MPH, Mason F. Lord Building, Center Tower, 5200 Eastern Ave., $7^{\text {th }}$ floor, Baltimore, MD 21224 (E-mail: ariel@jhmi.edu). provided care for people with dementia in the outpatient setting. We identified eligible participants through a central list of Johns Hopkins community physicians, an outpatient group practice in Maryland with 37 sites, and through personal contacts at other community-based practices. Most Johns Hopkins community physicians are full-time practitioners without academic appointments. We recruited participants electronically by using a combination of purposive, or expert, sampling and snowball sampling, in which participants recruited future participants from among their acquaintances. We focused on specialties where medications are frequently used to treat distressing symptoms of dementia or where medications are prescribed that may pose trade-offs for people with dementia: primary care, endocrinology, cardiology, and urogynecology.

\section{Data Collection and Analysis}

The interview guide (Appendix) was written by A.R.G., a geriatrician, and reviewed by C.C.G.C., a urogynecologist, and C.B., a geriatrician. We piloted the interview guide with 2 primary care clinicians and 1 urogynecologist and modified it based on the pilot testing. One investigator (A.R.G.) conducted the interviews in person or via video conference; a second investigator (P.L.) participated in interviews during July to August 2017. Interviews were audio-recorded and transcribed verbatim. Participant characteristics were collected using a questionnaire. Data collection continued until no new ideas were emerging.

We used constant comparison ${ }^{8,9}$ to analyze the content of the transcripts. Two team members, A.R.G. and P.L., independently read and coded 8 transcripts to create a codebook. Codes were based on existing literature ${ }^{4,10-13}$ about barriers to optimizing prescribing in older adults without dementia and on concepts that emerged from the transcripts. The codebook was applied to the remaining transcripts by P.L. and independently checked by A.R.G. Coding was discussed, and discrepancies were resolved through consensus. The coding was revised iteratively, and revisions were applied to all previously coded transcripts. Content analysis generated themes and subthemes. We used Atlas.ti, version 8, textual data analysis software (ATLAS.ti Scientific Software Development). 
Table 1. Participant Characteristics

\begin{tabular}{lc}
\hline Characteristic & Value \\
\hline Age, mean (SD) (years) & $46.6(9)$ \\
Race/ethnicity, n (\%) & \\
White & $10(48)$ \\
African American & $0(0)$ \\
Asian & $9(43)$ \\
Hispanic & $0(0)$ \\
Other & $2(10)$ \\
Academic degree, n (\%) & \\
MD & $18(86)$ \\
DO & $1(5)$ \\
CRNP & $2(10)$ \\
Specialty, n (\%) & \\
Family medicine & $6(29)$ \\
Internal medicine & $6(29)$ \\
Geriatric medicine & $3(14)$ \\
Urogynecology & $3(14)$ \\
Endocrinology & $3(14)$ \\
Cardiology & $3(14)$ \\
Clinic site, n (\%) & \\
Urban & $8(38)$ \\
Suburban & $9(43)$ \\
Rural/suburban & \\
Years since completing clinical training, & $4(19)$ \\
mean (SD) & $13.8(10)$ \\
No. of clinic sessions per week, mean (SD) & \\
proportion of patients with dementia in & \\
$<10 \%$ & $7.3(3)$ \\
$10 \%$ to $25 \%$ & \\
$26 \%$ to $75 \%$ & $0(02)$ \\
$>75 \%$ & $0(0)$ \\
\hline
\end{tabular}

SD, standard deviation.

*Participants could select more than one response.

${ }^{\dagger}$ The clinic served a mix of rural and suburban populations.

${ }^{\ddagger}$ One 4-hour session per week.

$\$$ As estimated by participant.

\section{Results}

Twenty-one clinicians from 16 different clinics participated (Table 1). Participants comprised 19 physicians and 2 nurse practitioners with a mean (SD) age of 47 (9) and a mean (SD) of 14 (10) years in practice. Content analysis revealed 3 major themes with subthemes.

\section{Theme 1: Clinician-Perceived Barriers to Optimizing Prescribing}

Clinicians described decisions about prescribing medicines in patients with dementia as complex. They described numerous barriers to optimizing prescribing in this population (Table 2). Some of these difficulties were unique to dementia, whereas others were heightened by the presence of dementia.

\section{Lack of Data}

Clinicians said they were challenged by the lack of predictive tools for life expectancy in people with dementia and the lack of clinical trial data on efficacy and safety of most medications for people with dementia. As a result, clinicians used "bedside gestalt" to assess life expectancy. Some worried about harming patients by stopping medications, such as statins or antihypertensive agents, and tended to continue preventive medications until patients reached advanced dementia. For example, 1 primary care clinician described continuing such medications as long as a patient was "pretty functional...Her blood pressure can go up and I do not want her to stroke out." The same clinician described another patient in whom she felt comfortable stopping such medications because the latter patient was "in a nursing home...not eating much...not communicative and...incontinent."

\section{Difficulty of Assessing Medication Effects in an Individual Patient}

Clinicians said it can be difficult to determine whether an individual patient with dementia is experiencing benefit or harm from a medication because the effects may be subtle or may develop insidiously or because a person with dementia may be unable to report symptoms. Some clinicians said they prescribed medications such as antidepressants for people with dementia but found it difficult to gauge effectiveness and wondered, "how much...we see what we want to see."

Others described using insensitive tools (eg, asking the patient, "Does a stone float?") to assess for cognitive adverse effects of medications and said that such assessment is "probably not realistic in a non-neurology practice." Some clinicians said that uncertainty about identifying adverse effects caused them to have a higher threshold for starting medications in people with dementia. Others relied on the caregiver's assessment of whether the patient was experiencing benefits or harms.

\section{Need to Consider Caregiver in Prescribing Decisions}

Another way in which dementia complicated prescribing decisions was that clinicians needed to 
Table 2. Clinician-Perceived Barriers to Optimizing Prescribing in Dementia

\begin{tabular}{|c|c|}
\hline Subtheme & Representative Quotation \\
\hline Lack of data & $\begin{array}{l}\text { Not only do I have to look at the evidence, but I also have to look at the whole } \\
\text { patient...I can't just focus on the guidelines... So many of these decisions are } \\
\text { subjective rather than objective. (primary care provider) }\end{array}$ \\
\hline $\begin{array}{l}\text { Difficulty of assessing medication effects in } \\
\text { an individual patient }\end{array}$ & $\begin{array}{l}\text { I would always do a little of the typical delirium check like, "Does a stone } \\
\text { float?" I often ask them, "Do you see anything you think isn't there or do } \\
\text { you hear anything you think isn't happening?" (primary care provider) }\end{array}$ \\
\hline $\begin{array}{l}\text { Need to consider caregiver availability, } \\
\text { knowledge, and skills in prescribing } \\
\text { decisions }\end{array}$ & $\begin{array}{l}\text { I have limited time with patients so I...really need to be selective about who I } \\
\text { end up talking to about [behavioral strategies to treat incontinence]. If it's } \\
\text { someone who's lucky to have someone even looking in on them once a day, } \\
\text { then what's the point of talking to them about that? (urogynecologist) }\end{array}$ \\
\hline $\begin{array}{l}\text { Perceptions of patient and caregiver beliefs } \\
\text { and expectations }\end{array}$ & $\begin{array}{l}\text { Caregivers worry about this decision means I gave up on mom or that I'm her } \\
\text { executioner because I stopped that med. (primary care provider) }\end{array}$ \\
\hline Cognitive biases & $\begin{array}{l}\text { I told the daughter, "I really don't see having this cholesterol lowering } \\
\text { medicine." It seemed like within maybe just } 2 \text { months, she went into the ER } \\
\text { and she had a stroke. (primary care provider) }\end{array}$ \\
\hline System barriers & $\begin{array}{l}\text { Maybe it is my place, but I'm not very good at calling another specialist and } \\
\text { saying, "I'm concerned about this. ." I'm the generalist. I'm not the } \\
\text { specialist. They know more than I do; this is their area. (primary care } \\
\text { provider) }\end{array}$ \\
\hline
\end{tabular}

consider the caregiver's availability and skills, particularly for decisions about prescribing anticoagulation, antihyperglycemic agents, and diuretics because of the serious harm that could occur with these medications. As 1 cardiologist said, "If it is a demented patient who needs anticoagulation but is living on their own...I have serious doubts about using it."

Although clinicians acknowledged the harms of polypharmacy, they generally perceived nonpharmacologic therapies as more onerous to patients and caregivers than medications. For example: "If it is a patient with a ton of comorbidities, including dementia, I think that can be a hard burden to...go to physical therapy on a weekly basis."

Clinicians described a tension between advocating for the patient and helping caregivers. Many said their prescribing decisions were influenced by the lack of nonpharmacologic resources to treat challenging behavioral symptoms of dementia, such as when caregivers struggled to cope with patients who were agitated. Clinicians said that in such scenarios, "We start Seroquel or Depakote whether they are effective or not...We cannot manage them [without medications]."

\section{Perceptions of Patient and Caregiver Beliefs and Expectations}

Perceived caregiver guilt was 1 of the most commonly cited factors that led clinicians to continue preventive medications, such as statins, even when they were unsure if the patient would benefit from them. Prescribing medications was often seen as "doing something for [the patient]," while stopping medications was seen as "giving up." Some clinicians said the belief that patients and caregivers were "expecting something" from them often led to a prescription.

\section{Cognitive Biases}

We found evidence of the availability heuristic, ${ }^{14}$ a mental shortcut that appeared to encourage prescribing or continuing medications to treat coexisting conditions for patients with dementia. For example, some clinicians recalled patients who had experienced negative outcomes after stopping a medication, such as a stroke occurring after discontinuation of a statin. Clinicians said such events deterred them from stopping patients' statins in the future.

Cognitive biases could also cause clinicians to withhold treatments, such as anticoagulation, from people with dementia. For example: "If someone has dementia, my perception would be that they are probably at higher risk for a bleeding event and that may or may not really be the case."

We also found evidence of therapeutic inertia, the tendency to continue prescribing drugs without periodic review of net benefit. ${ }^{15}$ As a cardiologist stated: "[Some clinicians] follow the path of least resistance...if nothing has changed and nothing is worse, do not stop anything. Just continue it be- 
cause there's no data against it, even though there may be no data for it."

\section{System Barriers}

Clinicians described numerous system barriers that they perceived as preventing them from optimizing prescribing for people with dementia. Many said they lacked time to discuss medications with patients and families or to communicate with other clinicians involved in a patient's care, particularly when the patient had providers in different health care systems. Primary care providers said they often accepted specialists' recommendations, even when they had doubts about whether a medication prescribed by the specialist was optimal for a patient with dementia. Other barriers cited by clinicians included clinician quality metrics that are not dementia specific (eg, hemoglobin A1c targets), and need to obtain prior authorization for medications that they believed were safer for patients with dementia, such as mirabegron instead of bladder antimuscarinics for treatment of incontinence.

\section{Theme 2: Perceptions of Enablers that May Help Optimize Prescribing}

Some clinicians felt more confident than others about their ability to optimize prescribing for people with dementia. These individuals described 4 enablers that they perceived as helping them to optimize prescribing: use of preprinted materials during office visits, such as flowcharts, to illustrate the risks and benefits of treatment options; diseasespecific clinical practice guidelines for nondementia illnesses that address the care of people with dementia; access to interdisciplinary services; and communication with other clinicians involved in a patient's care. The latter was perceived as helpful when clinicians were in the same health care system and could communicate through the electronic medical record; otherwise, communication was perceived as a barrier. The 2 perceived facilitators that were cited most often are described below:

\section{Clinical Practice Guidelines}

Endocrinologists repeatedly cited as helpful the American Diabetes Association (ADA) clinical practice recommendations, which address how cognitive function should affect hemoglobin A1c goals ${ }^{16}$ : "I keep the ADA standards of care table on my desk...A lot of times I will glance over and say, 'This lady has tons of comorbidities she has demen- tia. We're going to be happy with an A1C of 8.5 or 8 ,' and I write that in my note."

By contrast, other clinicians said that some clinical scenarios, such as whether or not to prescribe anticoagulation for stroke prevention in a patient with dementia and atrial fibrillation, were too "nebulous" to address in a guideline. As 1 cardiologist said, "Falling versus stroke or bleeding... These are not numbers you can follow, so it is much harder."

\section{Access to Interdisciplinary Expertise and Services}

Several clinicians said they benefited from having on-site pharmacists review patients' medications lists and provide guidance on tapering regimens or drug interactions. Other interdisciplinary services that were perceived as helpful included: on-site continence nurses or physiotherapists to teach lifestyle interventions or pelvic floor exercises (for patients with mild dementia) and social workers to help caregivers access resources or understand what to expect as dementia progresses. Access to such services was far from universal.

\section{Theme 3: Approaches to Discussing Medications}

Clinicians used a variety of approaches to discuss the risks and benefits of medications and to raise the issue of deprescribing with patients and caregivers (Table 3).

Some clinicians said they were explicit with caregivers that dementia is a life-limiting illness and that preventive medications should be stopped, although they varied in when during the course of dementia they made this transition or in what medications they considered essential. Others focused on the idea that stopping certain medications could enhance quality of life. Still other clinicians focused on long-term benefits of medications versus shortterm risks without explicitly mentioning life expectancy.

When asked to recall how they discuss the tradeoffs of medications with patients and caregivers, some clinicians described using subjective or conditional language. For example: "I would not say, 'This definitely causes harm. You have to stop it...' Are we worried that they are going to have more confusion? I will be honest with you, unless the patient or the family member specifically says they are concerned about it, I might not bring it up." In contrast, other clinicians recalled highlighting Food and Drug Administration warnings or using 
Table 3. Language Used by Clinicians to Discuss Medications and Deprescribing with Patients and Caregivers

\begin{tabular}{|c|c|}
\hline Subtheme & Representative Quotation \\
\hline Explicit mention of life expectancy & $\begin{array}{l}\text { I probably put it something like this: "Do you know that even if a person does } \\
\text { not have any medical issues, dementia by itself can shorten your life } \\
\text { expectancy? In your father or mother, the goal is to keep them comfortable. } \\
\text { With medications there is risk and what are we trying to achieve?" (primary } \\
\text { care provider) }\end{array}$ \\
\hline Focus on quality of life & $\begin{array}{l}\text { I broach it as a positive thing for the patient: "We're not harming them in any } \\
\text { way [by stopping medicines], but we're trying to give them a better quality } \\
\text { of life." (cardiologist) }\end{array}$ \\
\hline $\begin{array}{l}\text { Focus on long-term benefits of medication } \\
\text { versus short-term harms }\end{array}$ & $\begin{array}{l}\text { Someone like yourself with a lot of other medical problems, with maybe heart } \\
\text { problems, lung problems, memory problems, there probably is not a ton of } \\
\text { benefit to us being very aggressive in controlling your diabetes...I can } \\
\text { certainly get your blood sugars normal, but the problem is by doing that, I } \\
\text { expose you to a lot of risks. (endocrinologist) }\end{array}$ \\
\hline Conditional or subjective language & $\begin{array}{l}\text { I tell them the side effects of this medicine [bladder antimuscarinic] sometimes } \\
\text { are a dry mouth. It might cause a little memory problem. (primary care } \\
\text { clinician) }\end{array}$ \\
\hline Negative framing & $\begin{array}{l}\text { There are all these medications that you could try [for incontinence] but they } \\
\text { come with a significant risk of causing confusion. (primary care provider) }\end{array}$ \\
\hline
\end{tabular}

negative framing to emphasize potential harms of medications.

\section{Discussion}

We found that clinicians encountered numerous challenges to optimizing prescribing for patients with dementia, which likely explains why high rates of polypharmacy and PIM use persist in this population. Clinicians used a variety of strategies to discuss risks and benefits of medications with patients and caregivers and to raise the issue of deprescribing, suggesting that communication about optimal use of medications in people with dementia is an area of uncertainty.

A growing body of literature has identified barriers and facilitators of optimizing prescribing in older adults. This work has mainly focused on people without dementia, ${ }^{11,17}$ specific medication classes ${ }^{10}$ or PIMs, ${ }^{12}$ nursing home residents, ${ }^{18,19}$ or people near the end of life. ${ }^{20-22}$ Barriers include the lack of data to quantify benefits and harms, fear of adverse consequences, perceived patient and caregiver expectations, concern about being seen as abandoning the patient, and system barriers such as time constraints. Our findings show that there are similar issues when making prescribing decisions for people with dementia, and many of the barriers are heightened. For example, people with dementia are typically excluded from clinical trials. ${ }^{23}$ Life expectancy in dementia can vary between 3 to 10 years and can be difficult to predict. $^{24}$ As a result, clinicians in our study said they often have difficulty determining whether a medication is likely to benefit a patient with dementia during their remaining lifespan. Most clinical practice guidelines for nondementia illnesses do not address dementia, comorbidity, polypharmacy, or lack of evidence of efficacy for older adults. ${ }^{25}$ Recent guidelines for treatment of hypertension ${ }^{26}$ and diabetes ${ }^{16}$ recommend an individualized approach in people with dementia, yet considerable evidence gaps make this difficult for clinicians to implement. For example, little is known about the effects of antihypertensive treatment in patients with dementia ${ }^{26}$ or about how to facilitate shared decision making when there are competing health risks (eg, fall vs stroke). Future research is needed to provide data on benefits and harms of medications used to treat nondementia illnesses in people with various stages of dementia.

Clinicians reported that they often continue medications, including preventive ones, until patients reach advanced dementia. This is confirmed by quantitative evidence that patients, including people with dementia, continue to receive statins and other preventive medicines despite having limited life expectancy. ${ }^{27}$ Our findings are also consistent with a focus group study in Australia, in which clinicians expressed concern about stopping medications in patients with dementia and said they would feel more confident if they could provide patients and family caregivers with an educational resource that supported deprescribing "if there was back-up...evidence that [stopping a medication] was the appropriate thing to do." ${ }^{28}$ Some clinicians 
in our study said they continued medications as long as the patient was not experiencing adverse effects; yet, they also acknowledged the difficulty of identifying adverse effects in people with dementia who may not be able to report them. This tendency to continue medications is in contrast with the high levels of stress family caregivers report related to providing assistance with tasks related to medication management for a person with dementia, including obtaining refills, administering medications, and making judgements on the appropriateness of medications. ${ }^{29-32}$

We found that clinicians' prescribing decisions were heavily influenced by the availability, knowledge, and perceived expectations of caregivers. Clinicians said they sometimes offered medications with modest benefits and extensive risk profiles to treat distressing symptoms of dementia, including incontinence and agitation, because they viewed nonpharmacologic treatments as time consuming to explain or more burdensome to patients and caregivers than pharmacologic ones or because they felt they had no alternatives for caregivers. Nonpharmacologic interventions, such as structured activities, caregiver training, and comanagement with nonphysician care managers, have been shown to improve problem behaviors, reduce caregiver burden, improve patient and caregiver quality of life, or delay institutionalization. ${ }^{33-36}$ Yet, clinicians said that access to such services is poor. This may explain why education and support for nonpharmacologic strategies often take the form of a pamphlet from the clinician or the location of a support group. ${ }^{37}$

Clinicians reported using a variety of approaches to discuss medications with patients and families. Some clinicians used subjective or conditional language to discuss risks, for example, in describing bladder antimuscarinics: "[this medication] might cause a little memory problem," while others highlighted potential harms by citing research studies or Food and Drug Administration advisories: "There are all these medications that you could try [for incontinence] but they come with a significant risk of causing confusion." Clinicians also had varying approaches to discussing the concept of deprescribing or deintensifying treatment of coexisting conditions for patients with dementia. Little is known about the extent to which people with dementia and their caregivers recognize the trade-offs of medication use. For example, 1 recent qualitative study of caregivers suggests that they believe anti- psychotic medications to treat behavioral and psychological symptoms of dementia are generally safe, despite their well-documented potential for severe side effects. ${ }^{37}$ However, other qualitative studies reveal that older adults and caregivers experience tension between wanting to reduce medication use (due to burden and concerns about potential harms) and believing that their medications are necessary and beneficial. ${ }^{38,39}$

How best to foster informed decision making about medications in this population is currently unknown. Several communication strategies have shown evidence for improving patient understanding of the benefits and harms of treatment options in people without dementia. ${ }^{40}$ These include using plain language, presenting absolute risks using frequencies rather than relative risks, and making clear to patients the time interval over which a risk occurs. These and other approaches should be tested in people with dementia and their caregivers. Educational resources to help clinicians deprescribe unnecessary and PIMs in people with dementia are being developed. ${ }^{41,42}$ Knowledge of the barriers and facilitators identified in this study will be essential to future implementation of these resources. For example, educational materials and conversation guides could be created to help clinicians elicit patient and caregiver treatment goals at different stages of dementia and foster discussions about how dementia impacts the care of comorbid, nondementia illnesses. Such materials can be adapted from existing resources, such as the Medication Appropriateness Tool for Comorbid Health conditions during Dementia. ${ }^{43}$ To help ensure that patient goals guide treatment decisions, electronic health records should make patient goals readily visible to all clinicians across care settings. ${ }^{44} \mathrm{~Pa}-$ tient-directed materials can also be developed to help prime patients and caregivers for discussions about medication optimization in dementia. In addition, point-of-care risk prediction tools applicable to people with dementia could be developed to help clinicians, patients, and caregivers visualize the net benefit of specific medications (eg, anticoagulation for atrial fibrillation) as dementia progresses. Future research should investigate whether such tools positively impact outcomes that are important to patients with dementia and their caregivers.

Our study has some limitations. Qualitative interviews depend on the skill and assumptions of the interviewer. The study design inherently relied on 
self-report and our results may be prone to recall bias. Most participants practiced in urban or suburban settings and either had academic appointments or provided care at a community-based group practice that has an academic affiliation. We focused on a few specialties, and participants' experiences may not be generalizable. This study was not designed to be representative of all clinicians but rather to gain in-depth perspectives. It will be important for future research to seek the views and experiences of other stakeholders so that strategies for optimizing prescribing are inclusive of all patients with dementia, caregivers, and clinicians and adaptable to a variety of delivery systems and settings.

\section{Conclusions}

Despite the well-documented harms of polypharmacy and PIM use in people with dementia, clinicians face numerous barriers to optimizing prescribing in this population. Observational studies and trials should investigate the benefits and harms of stopping preventive medications in people at various stages of dementia. In addition, research should test different approaches for supporting informed decision making about medications by people with dementia and their caregivers. Lastly, the dissemination of effective nonpharmacologic approaches to care for people with dementia should be prioritized.

To see this article online, please go to: bttp://jabfm.org/content/ 32/3/383. full.

\section{References}

1. Alzheimer's Association. 2016 Alzheimer's disease facts and figures. Alzheimers Dement. 2016;12:459509.

2. Brauner DJ, Muir JC, Sachs GA. Treating nondementia illnesses in patients with dementia. JAMA 2000;283:3230-5.

3. Lin PJ, Zhong Y, Fillit HM, Chen E, Neumann PJ. Medicare expenditures of individuals with $\mathrm{Alz}$ heimer's disease and related dementias or mild cognitive impairment before and after diagnosis. J Am Geriatr Soc 2016;64:1549-57.

4. Reeve E, Bell JS, Hilmer SN. Barriers to optimising prescribing and deprescribing in older adults with dementia: a narrative review. Curr Clin Pharmacol 2015;10:168-77.

5. Lau DT, Mercaldo ND, Harris AT, Trittschuh E, Shega J, Weintraub S. Polypharmacy and potentially inappropriate medication use among communitydwelling elders with dementia. Alzheimer Dis Assoc Disord 2010;24:56-63.
6. Johnell K. Inappropriate drug use in people with cognitive impairment and dementia: a systematic review. Curr Clin Pharmacol 2015;10:178-84.

7. Palinkas LA, Horwitz SM, Green CA, Wisdom JP, Duan N, Hoagwood K. Purposeful sampling for qualitative data collection and analysis in mixed method implementation research. Adm Policy Ment Health 2015;42:533-44.

8. Glaser BG. The discovery of grounded theory: strategies for qualitative research. In: Strauss AL, ed. Chicago (IL): Aldine Pub. Co.; 1967.

9. Corbin JM. Basics of qualitative research techniques and procedures for developing grounded theory. In: Strauss AL, ed. Los Angeles (CA), SAGE; 2008.

10. Kouladjian L, Gnjidic D, Reeve E, Chen TF, Hilmer SN. Health care practitioners' perspectives on deprescribing anticholinergic and sedative medications in older adults. Ann Pharmacother 2016;50: 625-36.

11. Ailabouni NJ, Nishtala PS, Mangin D, Tordoff JM. General practitioners' insight into deprescribing for the multimorbid older individual: a qualitative study. Int J Clin Pract 2016;70:261-76.

12. Anderson K, Stowasser D, Freeman C, Scott I. Prescriber barriers and enablers to minimising potentially inappropriate medications in adults: a systematic review and thematic synthesis. BMJ Open 2014; 4:e006544.

13. Cook JM, Marshall R, Masci C, Coyne JC. Physicians' perspectives on prescribing benzodiazepines for older adults: a qualitative study. J Gen Intern Med 2007;22:303-7.

14. Croskerry P. The importance of cognitive errors in diagnosis and strategies to minimize them. Acad Med 2003;78:775-80.

15. Gurwitz JH. The physics of geriatric pharmacotherapy: overcoming therapeutic inertia and momentum. Am J Med 2012;125:523-4.

16. American Diabetes Association. 11. Older adults: standards of med care in diabetes-2018. Diabetes Care. 2018;41:S119-S25.

17. Schuling J, Gebben H, Veehof LJ, HaaijerRuskamp FM. Deprescribing medication in very elderly patients with multimorbidity: the view of Dutch GPs. A qualitative study. BMC Fam Pract 2012;13:56.

18. Palagyi A, Keay L, Harper J, Potter J, Lindley RI. Barricades and brickwalls-a qualitative study exploring perceptions of medication use and deprescribing in long-term care. BMC Geriatr 2016;16:15.

19. Turner JP, Edwards S, Stanners M, Shakib S, Bell JS. What factors are important for deprescribing in Australian long-term care facilities? Perspectives of residents and health professionals. BMJ Open 2016; 6:e009781.

20. Holmes HM, Hayley D, Alexander G, Sachs GA. Reconsidering medication appropriateness for patients late in life. Arch Intern Med 2006;166:605-9. 
21. Narayan SW, Nishtala PS. Discontinuation of preventive medicines in older people with limited life expectancy: a systematic review. Drugs Aging 2017; 34:767-76.

22. Parsons C, Hughes CM, Passmore AP, Lapane KL. Withholding, discontinuing and withdrawing medications in dementia patients at the end of life: a neglected problem in the disadvantaged dying? Drugs Aging 2010;27:435-49.

23. Zulman DM, Sussman JB, Chen X, Cigolle CT, Blaum CS, Hayward RA. Examining the evidence: a systematic review of the inclusion and analysis of older adults in randomized controlled trials. J Gen Intern Med 2011;26:783-90.

24. Zanetti O, Solerte SB, Cantoni F. Life expectancy in Alzheimer's disease (AD). Arch Gerontol Geriatr 2009;49:237-243.

25. Boyd CM, Darer J, Boult C, Fried LP, Boult L, Wu AW. Clinical practice guidelines and quality of care for older patients with multiple comorbid diseases: implications for pay for performance. JAMA 2005; 294:716-24.

26. Whelton PK, Carey RM, Aronow WS, et al. 2017 ACC/AHA/AAPA/ABC/ACPM/AGS/APhA/ASH/ ASPC/NMA/PCNA guideline for the prevention, detection, evaluation, and management of high blood pressure in adults. A report of the american college of cardiology/american heart association task force on clinical practice guidelines. Hypertension 2018;71:e127-e248.

27. Poudel A, Yates P, Rowett D, Nissen LM. Use of preventive medication in patients with limited life expectancy: a systematic review. J Pain Symptom Manage 2017;53:1097-1110.e1091.

28. Page AT, Clifford RM, Potter K, et al. Exploring the enablers and barriers to implementing the Medication Appropriateness Tool for Comorbid Health conditions during Dementia (MATCH-D) criteria in Australia: a qualitative study. BMJ Open 2017;7: e017906.

29. Smith F, Grijseels MS, Ryan P, Tobiansky R. Assisting people with dementia with their medicines: experiences of family carers. Int J Pharm Pract 2015; 23:44-51.

30. Wolff JL, Spillman BC, Freedman VA, Kasper JD. A national profile of family and unpaid caregivers who assist older adults with health care activities. JAMA Intern Med 2016;176:372-9.

31. Kasper JD, Freedman VA, Spillman BC, Wolff JL. The disproportionate impact of dementia on family and unpaid caregiving to older adults. Health Aff (Millwood) 2015;34:1642-9.
32. Reinhard SC, Levine C, Samis S. Home alone: family caregivers providing complex chronic care. Washington, D.C., AARP; 2012.

33. Gitlin LN, Kales HC, Lyketsos CG. Nonpharmacologic management of behavioral symptoms in dementia. JAMA 2012;308:2020-9.

34. Callahan CM, Boustani MA, Unverzagt FW, et al. Effectiveness of collaborative care for older adults with Alzheimer disease in primary care: a randomized controlled trial. JAMA 2006;295:2148-57.

35. Jennings LA, Tan Z, Wenger NS, et al. Quality of care provided by a comprehensive dementia care comanagement program. J Am Geriatr Soc 2016;64: 1724-30.

36. Bass DM, Judge KS, Snow AL, et al. Caregiver outcomes of partners in dementia care: effect of a care coordination program for veterans with dementia and their family members and friends. J Am Geriatr Soc 2013;61:1377-86.

37. Kerns JW, Winter JD, Winter KM, Kerns CC, Etz RS. Caregiver perspectives about using antipsychotics and other medications for symptoms of dementia. Gerontologist. 2018;58:e35-e45.

38. Reeve E, Low LF, Hilmer SN. Beliefs and attitudes of older adults and carers about deprescribing of medications: a qualitative focus group study. $\mathrm{Br} \mathrm{J}$ Gen Pract 2016;66:e552-560.

39. Moen J, Bohm A, Tillenius T, Antonov K, Nilsson JL, Ring L. "I don't know how many of these [medicines] are necessary." - a focus group study among elderly users of multiple medicines. Patient Educ Couns 2009;74:135-41.

40. Fagerlin A, Zikmund-Fisher BJ, Ubel PA. Helping patients decide: ten steps to better risk communication. J Natl Cancer Inst 2011;103:1436-43.

41. Farrell B, Black C, Thompson W, et al. Deprescribing antihyperglycemic agents in older persons: evidence-based clinical practice guideline. Can Fam Physician 2017;63:832-43.

42. Jennings AA, Boyle S, Foley T. The development and evaluation of an online dementia resource for primary care based health professionals. Internet Interv 2018;11:47-52.

43. Page AT, Potter K, Clifford R, McLachlan AJ, Etherton-Beer C. Medication appropriateness tool for co-morbid health conditions in dementia: consensus recommendations from a multidisciplinary expert panel. Intern Med J 2016;46:1189-97.

44. Nagykaldi ZJ, Tange H, De Maeseneer J. Moving from problem-oriented to goal-directed health records. Ann Fam Med 2018;16:155-9. 
Appendix. Interview Guide for Primary Care Providers and Specialists

Opening Question: Decision-Making Process

Please think back to an older patient that you have recently seen with dementia and other chronic conditions.

- How did you make a decision to prescribe or continue a medication to treat this patient's comorbid chronic condition? Walk me through the steps.

- Probes if they have difficulty thinking of an example: Warfarin or other oral anticoagulants for afib, blood pressure or lipid-lowering medications, diabetic agents, bladder antimuscarinics for overactive bladder.

\section{Factors Influencing Decision Making}

- Was the decision different because the patient had dementia than it would have been in another 1 of your patients? How do you see dementia as either similar or different to other conditions?

- Were there any particular trade-offs you considered in this patient because of their dementia?

- In general, what factors do you consider when prescribing for patients with dementia?

- Probes: How did the patient's family influence your prescribing decision? How did the patient's place of residence (home vs assisted living) influence your prescribing decision? How heavily did you weigh clinical practice guidelines?

- [The next three questions are for primary care clinicians only] Did you refer the patient to a specialist for this problem? Why (or why not)?

- Probes: If so, how influential was the specialist in your decision about whether to prescribe (or continue) the medication?

- Do you typically communicate with specialists who are caring for your patients with dementia about prescribing decisions? If so, how do you communicate with them?

- Have you ever had a concern about a medicine that another clinician prescribed to a patient with dementia? If so, what did you do?

\section{Knowledge and Skills: Discussing Trade-Offs}

- You mentioned some trade-offs that you considered in this patient because of their dementia. How did you discuss these trade-offs with the patient or their family? What did you say? What phrases did you use?

\section{Knowledge and Skills: Identifying PIMs}

- Are there any medicines or classes of medicines that are commonly used [for specialists add, "in your specialty"] that you consider potentially inappropriate or harmful in older patients with dementia? If so, which ones? Why?

- Probe: Are there any resources or tools you use to determine whether a medicine is potentially inappropriate or harmful in people with dementia?

\section{Barriers and Facilitators of High-Quality Prescribing Decisions}

- In general, do you feel comfortable making prescribing decisions for patients with dementia?

- Is there anything that helps make it easier to make prescribing decisions for patients with dementia?

- Do you encounter any barriers that make it difficult for you to make prescribing decisions for patients with dementia?

- What would help you feel more comfortable making prescribing decisions for patients with dementia?

\section{For Specialists Only: Communication with Primary Care Physicians}

- Do you routinely communicate with the patient's primary care physician about prescribing decisions for patients with dementia? If so, how?

\section{For Urogynecologists Only}

- Are there nonpharmacologic or other strategies that you use to manage incontinence in patients with dementia? Tell me about them.

- How well do they work? How accessible are they to your patients and their families? 